\title{
Evaluation of damping estimates by automated Operational Modal Analysis for offshore wind turbine tower vibrations
}

\author{
Bajri, Anela; Høgsberg, Jan Becker; Rüdinger, Finn
}

Published in:

Renewable Energy

Link to article, DOI:

10.1016/j.renene.2017.03.043

Publication date:

2018

Document Version

Peer reviewed version

Link back to DTU Orbit

Citation (APA):

Bajri, A., Høgsberg, J. B., \& Rüdinger, F. (2018). Evaluation of damping estimates by automated Operational Modal Analysis for offshore wind turbine tower vibrations. Renewable Energy, 116, 153-163.

https://doi.org/10.1016/j.renene.2017.03.043

\section{General rights}

Copyright and moral rights for the publications made accessible in the public portal are retained by the authors and/or other copyright owners and it is a condition of accessing publications that users recognise and abide by the legal requirements associated with these rights.

- Users may download and print one copy of any publication from the public portal for the purpose of private study or research.

- You may not further distribute the material or use it for any profit-making activity or commercial gain

- You may freely distribute the URL identifying the publication in the public portal 


\title{
Evaluation of damping estimates by automated Operational Modal Analysis for offshore wind turbine tower vibrations
}

\author{
Anela Bajrića, a $^{\mathrm{a}}$ Jan Høgsberg ${ }^{\mathrm{a}}$, Finn Rüdinger ${ }^{\mathrm{b}}$ \\ ${ }^{a}$ Technical University of Denmark, DTU Mechanical Engineering, Nils Koppels Allé Building 403, 2800 Kgs. \\ Lyngby, Denmark \\ ${ }^{b}$ DONG Energy, Wind Power, LAC Department, Nesa Allé 1, 2820 Gentofte, Denmark
}

\begin{abstract}
Reliable predictions of the lifetime of offshore wind turbine structures are influenced by the limited knowledge concerning the inherent level of damping during downtime. Error measures and a automated procedure for covariance driven Operational Modal Analysis (OMA) techniques has been proposed with a particular focus on damping estimation of wind turbine towers. In the design of offshore structures the estimates of damping are crucial for tuning of the numerical model. The errors of damping estimates are evaluated from simulated tower response of an aeroelastic model of an $8 \mathrm{MW}$ offshore wind turbine. In order to obtain algorithmic independent answers, three identification techniques are compared: Eigensystem Realization Algorithm (ERA), covariance driven Stochastic Subspace Identification (COV-SSI) and the Enhanced Frequency Domain Decomposition (EFDD). Discrepancies between automated identification techniques are discussed and illustrated with respect to signal noise, measurement time, vibration amplitudes and stationarity of the ambient response. The best bias-variance error trade-off of damping estimates is obtained by the COV-SSI. The proposed automated procedure is validated by real vibration measurements of an offshore wind turbine in non-operating conditions from a 24-hour monitoring period.
\end{abstract}

Keywords: Offshore wind turbine, Ambient tower vibrations, Correlation function estimators, Automated Operational Modal Analysis, Damping estimation

\footnotetext{
${ }^{*}$ Corresponding author

Email address: abaj@mek.dtu.dk (Anela Bajrić )
} 


\section{Introduction}

Fatigue damage accumulation during the lifetime of an offshore wind turbine generator is strongly dependent on the damping during downtime. Interpretation and analysis of the monitoring data by new real-time fault diagnosis and fault-tolerant control techniques can help the designer to improve the system reliability [1] and minimize downtime caused by malfunctioning equipment. As the wind farms are being developed at increasing water depths the transportation time from harbor to site increases, and a reduction of downtime hours is therefore not expected.

Cost effective and reliable structural designs of wind turbines are strongly dependent on the accuracy of the employed damping model. However, the system-damping matrix is often based on naive assumptions of energy dissipation phenomena. Therefore, reliable damping estimates are crucial for tuning or improving the mathematical representation of damping. In order to achieve cost effective and reliable structural designs an accurate and precise procedure for estimation of the damping is a necessity. In this paper, the reliability is sought by achieving estimates of viscous damping independent of the chosen OMA algorithm.

The vibration amplitude is governed by the amount of inherent damping present in the structures. The main sources of damping are associated with the structure, the aerodynamic and hydrodynamic interaction and the deformation of the soil by the foundation, and in some cases additional damping is provided by an external damper. Two conditions may in particular be limiting factors in the design of offshore wind turbine foundations, due to low levels of damping. The first condition is due to misaligned wind and wave loading on the structure, which cause low damping associated with sideside vibrations, while in operating conditions [2]. The second condition occurs during non-operating conditions, in which the total damping will be entirely governed by the small amount of structural and foundation damping, while the otherwise significant contribution from aerodynamic interaction is negligible. The damping in the fore-aft direction is particularly critical, as the blades are pitched out. Accurate estimation of the inherent damping is therefore an important design parameter for the next generation of offshore wind turbine structures.

Damping has traditionally been estimated from the free decay response obtained from rotor stop tests, which is sensitive to the environmental conditions [3]. OMA enables modal identification based 
on ambient vibrations. In the last decade, OMA has drawn attention in connection with offshore wind turbines [5-10], despite the restrictive assumptions which these algorithms have to comply with, i.e. white noise input and linear time-invariant system. During non-operating conditions of wind turbines the vibration levels are relatively low and the assumption of a linear time-invariant system is acceptable. On the other hand, if more complex and nonlinear behavior is observed, data data-driven approaches are preferable to estimate the underlying system model [10]. Within the OMA framework, damping estimates often exhibit high variability causing skepticism concerning the physical interpretation of the results [11]. The difficulties encountered when trying to estimate damping from ambient vibrations of wind turbines has been discussed in [12]. Firstly, the successful estimation of damping depends on how realistic the underlying mathematical model of the estimation technique is. Additionally the employed procedure for automated identification also has an influence on the quality of the estimation of modal parameters. Several identification techniques have been compared, evaluated and automated for the estimation of mode shapes and frequencies, see for instance [14-20]. However there is a lack of benchmark techniques concerning the estimation of damping in offshore wind turbines, and evaluation of sources of errors related to the existing techniques which influence the quality of the estimates.

In the present paper an automated identification procedure is proposed, together with evaluation measures which indicate the type of error in the estimates of damping. To ensure algorithm independent answers three existing techniques are automated and evaluated: the ERA, the COV-SSI and the EFDD. The automated identification procedure proposed for techniques in the time domain determines the number of time lags to include in the estimation and the model order.

The variations in the estimates of damping obtained by the automated procedure are initially presented through two numerical case studies and finally applied to measured ambient vibrations of an offshore wind turbine. The first numerical study is a Monte Carlo simulation of the random response of a representative two degree-of-freedom (2DOF) system. Preliminary discrepancies in the estimates of damping are found to originate from the choice of technique and pre-processing of the response. Subsequently, the damping estimates obtained by the automated procedure are assessed for simulated offshore wind turbine tower vibrations, where the fluctuations in the tower response 
measurements are deliberately contaminated by signal noise. The automated procedure of damping estimation is finally demonstrated for a real offshore wind turbine in non-operating conditions from a 24-hour monitoring period. From the tower top vibrations the automated procedure is able to identify the low levels of the damping in the fore-aft mode compared to the side-side mode in nonoperating conditions. An extensive discussion concerning the low damping levels and improvements of the estimation techniques is provided.

\section{Damping estimation from ambient vibrations}

The time domain methods, ERA and COV-SSI, have been impremented following their original formulation [20, 21]. An extensive review of OMA is provided in [22]. The ERA is an extension to Multiple-Input-Multiple-Output (MIMO) systems of the Ibrahim Time Domain (ITD) identification $[23,24]$. The data driven SSI has been shown to have properties similar to those of the COV-SSI [25] and the data driven SSI is therefore not considered in the present analysis. The objective of both time domain techniques is to estimate a discrete-time system matrix. The eigenvalue decomposition of the system matrix is interpreted as a set of discrete complex system poles. The continuous time poles are then determined as,

$$
\lambda=\frac{\ln (\mu)}{\Delta t}
$$

where $\mu$ is the discrete time pole, and $\Delta t$ is the time increment. The continuous poles can be related to the natural frequency and the damping of a mode of the system,

$$
\lambda=-\omega \zeta \pm i \omega_{D}
$$

where $i$ is the imaginary unit, $\omega_{D}=\omega \sqrt{1-\zeta^{2}}$ is the damped natural angular frequency, $\omega$ is the undamped natural angular frequency and $\zeta$ is the modal damping ratio. Hence, the total damping contribution is estimated by the proposed techniques. The natural frequency is thus obtained as $f=\omega / 2 \pi$. The eigenvectors of the discrete system matrix are interpreted as the mode shapes, which are transformed to the physical coordinates by the observation matrix.

The only frequency domain technique considered in the present study is the EFDD [26], which is an adaption of the Complex Mode Indicator Function (CMIF) [27]. All the three chosen identification 
algorithms rely on the evaluation of the estimated correlation matrix. Such pre-processing will inevitably introduces estimation errors that propagate through the identification procedure, and in particular affect the resulting damping estimates.

\subsection{Measures of performance for evaluation of estimation errors}

To assess the quality of an estimate, three performance measures have been proposed. An approximation of a quantity will in the following be referred to as an estimate, while a parameter estimator will be identified by its given name, as for example ERA. Consider $\hat{\theta}$ as an estimate of $\theta$, based on $N$ samples of a random process. The quality of the estimate or estimator is validated in terms of the bias $(B)$, the variance $(V)$ and the mean squared error (MSE) of the realization. These are defined as,

$$
\begin{aligned}
B(\hat{\theta}, \theta) & =E[\hat{\theta}]-\theta \\
V(\hat{\theta}) & =E\left[(\hat{\theta}-E[\hat{\theta}])^{2}\right] \\
\operatorname{MSE}(\hat{\theta}, \theta) & =E\left[(\hat{\theta}-\theta)^{2}\right]=B(\hat{\theta}, \theta)^{2}+V(\hat{\theta})
\end{aligned}
$$

where $E[\cdot]$ is the expectation operator. The variance and mean squared error are unfortunately associated with large weighting outliers due to the squaring of each term.

\subsection{Pre-processing in the time domain}

Bias and variance in the damping estimates using correlation driven OMA techniques have been attributed to the inclusion of the tail regions in the correlation function estimates [14]. This issue is addressed in the following. Variability on the choice of correlation function estimate has been found in literature $[22,28]$. Therefore, two types of correlation function estimates have been considered: an unbiased and a biased estimate. The general expression of the correlation function for ergodic wide-sense stationary processes $X(t)$ and $Y(t)$ is

$$
R_{x y}(\tau)=E[X(t) Y(t+\tau)]=\lim _{T \rightarrow \infty} \frac{1}{T} \int_{t=0}^{T} x(t) y(t+\tau) d t
$$


for $0 \leq \tau<T$, where $\tau$ is the time separation and $T$ is the total period of the realizations $x(t)$ and $y(t)$. For zero mean Gaussian processes the covariance function will be equivalent to the correlation function, and the latter is therefore used in the following.

Two truncations of (4) are widely used to approximate the correlation function for finite realizations. These are referred to as the unbiased and biased estimate of the correlation function and are in the following distinguished by $\left({ }^{\wedge}\right)$ and $\left(^{-}\right)$, respectively. The unbiased correlation function estimate for positive time lags is given by

$$
\hat{R}_{x y}(m)=\frac{1}{N-m} \sum_{n=0}^{N-1-m} x(n) y(n+m)
$$

for $n=m=0,1,2, \ldots, N-1$, where $x(n)$ and $y(n)$ are the discrete representation of the realizations $x(t)$ and $y(t)$ at time increment $n$, respectively. The discrete representation in (5) is related to the continuous representation by $t=n \Delta t, \tau=m \Delta t$ and $T=(N-1) \Delta t$. The bias and variance of an estimator can be computed by the relations in (3), which have been derived in [29] for the correlation function estimators. Evaluating the bias for $(5)$ gives $B\left(\hat{R}_{x y}(m)\right)=0$, hence the name unbiased correlation function estimator. The variance of (5) can be approximated as

$$
V\left(\hat{R}_{x y}(m)\right) \approx \frac{N}{(N-m)^{2}} \sum_{n=-\infty}^{\infty}\left[R_{x x}(n) R_{y y}(n)+R_{x y}(n-m) R_{y x}(n+m)\right]
$$

where $R_{x x}$ and $R_{y y}$ are the auto-correlations of the processes $x$ and $y$, while $R_{x y}$ and $R_{y x}$ are the corresponding cross-correlations. When $m \rightarrow N$, the variance of the unbiased correlation function estimate will increase, and the so called tail regions will be erroneous. An alternative truncation of (4) can therefore be expressed as

$$
\bar{R}_{x y}(m)=\frac{1}{N} \sum_{n=0}^{N-1-m} x(n) y(n+m)
$$

The bias of this estimator is

$$
B\left(\bar{R}_{x y}(n)\right)=-\frac{m}{N} R_{x y}(m)
$$

and thus (7) is denoted as the biased estimator of the correlation function. However, the bias is seen to vanish as $N \rightarrow \infty$, and therefore (7) is asymptotically unbiased. Furthermore, the variance of (7) 
can be approximated by

$$
V\left(\bar{R}_{x y}(m)\right) \approx \frac{1}{N} \sum_{n=-\infty}^{\infty}\left[R_{x x}(n) R_{y y}(n)+R_{x y}(n-m) R_{y x}(n+m)\right]
$$

from which it is evident that the variance of (7) converges to zero when $N \rightarrow \infty$. This property is also valid for the estimator in (5), hence the biased and unbiased correlation function estimator are both consistent. The best choice of correlation function estimator is determined by a trade-off between bias and variance error, also known as the bias-variance dilemma [29]. The influence of the choice of correlation function estimator with respect to the damping estimation is discussed in Section 3 .

\subsection{Pre-processing in the frequency domain}

Estimation errors are present in the spectral density estimate of a stochastic response due to finite response measurements and the frequency resolution. The bias error in the estimation of the spectral density using a Hanning window was studied in detail in [30, 31]. For the estimation of damping it is important to have a sufficiently high frequency resolution relative to the bandwidth ratio. This can be expressed as

$$
\Delta f=\frac{1}{N \Delta t} \quad, \quad B_{r} \approx 2 \zeta f
$$

where $\Delta f$ is the sampling frequency and $B_{r}$ is the half-power bandwidth for light damping at the resonance frequency. The ratio $B_{r} / \Delta f$ therefore controls the bias error, such that less bias error is introduced by a larger ratio. The bias error may result in erroneous damping estimates, which was studied in $[32,33]$ and will not be further addressed in the present paper.

\subsection{Automated identification}

Effective automated estimation of damping can provide substantial information to tune damping models for the design of offshore wind turbines. Automated identification in the frequency domain is implemented according to [34], and will not be further discussed. The automate procedure presented in this paper is suitable for correlation driven OMA techniques, focusing on natural frequency and damping estimation. The procedure can be extended to include automated identification of 
mode shapes by considering the Modal Assurance Criterion (MAC) [35]. In this study, the focus is mainly on reliable automated estimation of natural frequency and damping, and the MAC value will therefore not be further discussed. The procedure consists of three main steps categorized as: the preliminary sorting of poles, the stabilization of poles and evaluation of the residual sum of squares. The two first steps are common practice in OMA [18, 19], and these two are therefore only briefly described.

\subsubsection{Preliminary sorting of poles.}

The first step in the automated procedure concerns the removal of the unwanted continuous complex poles $\lambda$ obtained for the $n$-order realization of the dynamic matrix. The initial removal procedure is based on the following two basic criteria,

$$
\zeta \leq 0 \quad, \quad \omega_{D}=0
$$

where the first criterion removes poles with a positive real part, associated with negative damping, while the second criterion removes non-oscillatory poles that do not appear in complex conjugate pairs.

\subsubsection{Stabilization of poles.}

The stabilization criteria typically identify a cluster of stabilized poles for specific thresholds of the relative difference in the natural angular frequency $\omega$, or damping $\zeta$ estimated at model order $j$ relative to model order $h$ by the expressions

$$
d \omega_{h, j}=\frac{\left|\omega_{h}-\omega_{j}\right|}{\max \left(\omega_{h}, \omega_{j}\right)}, d \zeta_{h, j}=\frac{\left|\zeta_{h}-\zeta_{j}\right|}{\max \left(\zeta_{h}, \zeta_{j}\right)}
$$

A cluster of stabilized poles is identified by the differences $d \omega_{h, h+1}$ and $d \zeta_{h, h+1}$ between two adjacent model orders $h$ and $j=h+1$ for a predefined threshold value of $d \omega_{h, j}$ and $d \zeta_{h, j}$. Subsequently, the angular frequency and damping ratio are evaluated from the complex poles in each of the identified clusters based on the automated procedure described in the following. 


\subsubsection{Automated evaluation by the residual sum of squares.}

The final step of the automated procedure is the selection of the number of time lags and model order. The procedure is based on the reconstruction of the auto-correlation function from the cluster of complex poles. The reconstruction of the auto-correlation function is now presented, while the procedure for obtaining the optimal choice of lags and model order is described subsequently. The auto-correlation function of an underdamped SDOF system excited by white noise corresponds to [36]

$$
R_{x x}(\tau)=\frac{\pi \omega S_{0}}{2 k^{2} \zeta}\left(\cos \left(\omega_{D} \tau\right)+\frac{\zeta}{\sqrt{1-\zeta^{2}}} \sin \left(\omega_{D} \tau\right)\right) \exp (-\omega \zeta \tau), \quad \tau>0
$$

where $k$ is the structural stiffness and $S_{0}$ is the constant power spectral density of the white noise excitation. The envelope of the auto-correlation function in (13) is

$$
z_{R_{x x}}(\tau)=A \exp (-\omega \zeta \tau)
$$

with initial magnitude $A=\pi \omega S_{0} /\left(2 k^{2} \zeta\right)$. Thus, the auto-correlation function of an SDOF system excited by white noise, is equivalent to the impulse response function of a lightly damped SDOF system. This is the fundamental assumption of the proposed automated procedure.

The estimated frequency and damping in the cluster of stabilized complex poles obtained by (12) can be evaluated by the fit of the envelope of the impulse response function in (14) with the envelope of the estimated auto-correlation function in (5) or (7) obtained by the random vibration signals. The optimal estimate of the frequency and damping in the cluster of stabilized poles is found by minimizing the residual sum of squares for a unit magnitude $(A=1)$,

$$
R S S=\sum_{m}\left(z_{R_{x x}}(m)-z_{\hat{R}_{x x}}(m)\right)^{2}
$$

where $z_{\hat{R}_{x x}}(m)$ is the envelope of the estimated auto-correlation function determined by either $(5)$ or (7).

The expression of the residual sum of squares in (15) does not contain the model order explicitly. Each complex pole is a function of the model order, and the pole forms the product $\zeta \omega$ through the relation in (2). A cluster of poles is formed for each time separation $\tau=m \Delta t$, where $T$ subsequently determines the size of the block-Hankel and block-Toeplitz matrices. This implies that the residual 
sum of squares given in (15) must be computed for clusters of poles for each time separation $m$. The optimal estimate of modal parameters is the one which yields the minimum residual sum of squares.

\section{Automated damping estimation in $2 \mathrm{DOF}$ system}

The damping estimates are constant for a viscously damped 2DOF spring-mass systems, and the model errors are of negligible magnitude [37]. Therefore Monte Carlo simulations have been performed to emphasize the variations introduced by finite realizations of a stochastic process for a system with representative properties. The dynamic response has been simulated for the viscously damped 2DOF spring-mass system shown in Figure 1, with natural frequencies $f_{1}=0.2286 \mathrm{~Hz}$ and $f_{2}=1.2031 \mathrm{~Hz}$ and corresponding modal damping ratios $\zeta_{1}=0.0127$ and $\zeta_{2}=0.0308$ for the two modes. These properties are representative of the off-shore wind turbine considered in Section 4 and Section 5. The system has been excited by a white noise process, obtained from a normal distribution of pseudo random numbers generated in Matlab. The acceleration time histories for both structure masses have been obtained by the Newmark time integration scheme with $\beta=\frac{1}{4}$ and $\gamma=\frac{1}{2}$. The length of each stochastic time history is 4 hours with a time increment of $\Delta t=0.1 \mathrm{~s}$. The simulation length is therefore equivalent to 3300 vibration periods of the first vibration mode.

The computation of the unbiased and biased correlation functions has been performed for positive time lags, and based on the acceleration records without any normalization. The normalization required to obtain the unbiased and biased estimate has been performed by the expressions in (5) and (7), such that both estimates are comparable because they originate from the same underlying process. The estimated Power Spectral Density (PSD) has been computed using Welch's method of spectral estimation, applying a Hanning window for the averaging with an overlap of $50 \%$. The estimation is based on $2^{18}$ sampling points, whereby the bandwidth to frequency resolution ratio becomes greater than 11.5, satisfying the criterion for a bias error in the PSD of less than 1\% [30]. 


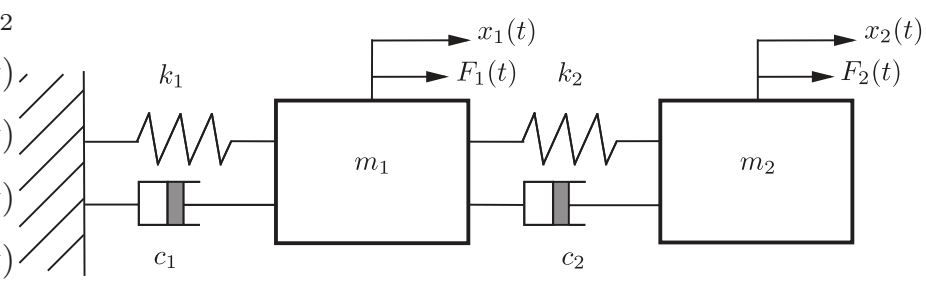

Figure 1: Viscously damped 2DOF spring-mass system.

The pre-processed signal has been used as input to the three identification techniques, namely ERA, COV-SSI and EFDD. Two clusters of stabilized poles have been found by the stabilization criteria in (12) set to $0.5 \%$ for both the relative difference in frequency and damping. The first ten periods of the respective auto-correlation function of the mode of interest has been used to evaluate the minimum residual sum of squares in (15), thereby determining the model order and the size of the block-Hankel and block-Toeplitz matrix for the estimation techniques in the time domain. A model order of four has consistently been determined, which is as expected for a 2DOF system. The number of time lags which resulted in the minimum residual sum of squares fluctuated around 500 for the first mode, corresponding to 11 vibration periods. The auto-correlation function, for the uncoupled modes, obtained by the EFDD has been used to estimate the damping ratio using the log decrement procedure, where the first ten periods were considered. The identification procedure has been repeated 100 times, and the statistical properties are the basis for the evaluation of the estimates of the damping.

The distributions of the estimated damping of the first mode are shown in Figure 2. A distinct difference is observed between the estimates obtained by the time domain techniques, ERA and COV-SSI, and those obtained by EFDD. The right tail of the distribution obtained by the EFDD is in particular noticeable, indicating that this technique leads to a positively skewed distribution of the damping estimates. The distributions for the damping estimates of the second mode, shows similar tendencies and are therefore not presented. 


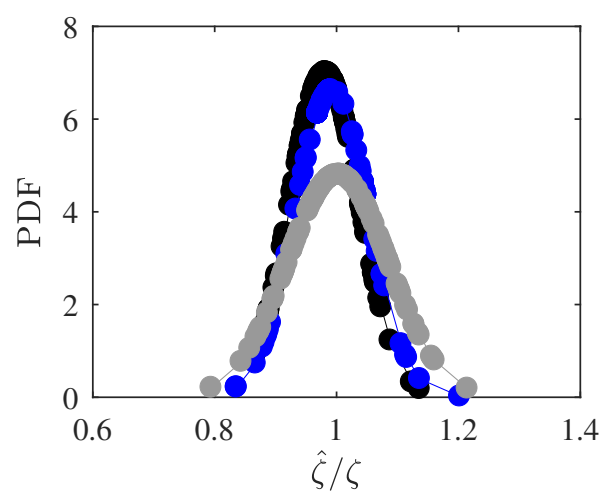

Figure 2: Estimate of the damping of the first mode of a 2DOF system obtained by EFDD (grey bullet), and from from unbiased correlation function estimates by COV-SSI (blue bullet), and ERA (black triangle).

Table 1 shows that the EFDD yields positive bias for damping, and the greatest variance and mean squared error in comparison to the ERA and COV-SSI. The variance and mean squared error of the ERA and COV-SSI exhibit minor differences. The bias is negative, which shows that the damping is underestimated by the time domain techniques, with a larger bias for the ERA than the COV-SSI method. The overall performance of the identification indicates that the COV-SSI seems to be the most effective technique in estimating the damping.

The differences between the various techniques in estimating damping for post-processing by the biased correlation function in comparison to the unbiased correlation function are minor. Therefore the distribution of the estimates by the biased correlation function is not presented, however the bias, variance and mean squared error are given in Table 1. The mean squared error is smallest for post-processing by the unbiased correlation function. The errors in the estimate of the natural frequencies are given in Table 1, which shows that the variance and subsequently the mean squared error are three orders of magnitude smaller than those for the damping estimates. 
Table 1: The bias, variance and mean squared error of the damping and frequency of the first mode of the 2DOF system. The unbiased, biased correlation function and power spectral density are the pre-processing variations for identification techniques, ERA, SSI and EFDD.

\begin{tabular}{|c|c|c|c|c|c|}
\hline & \multicolumn{2}{|c|}{ Unbiased correlation } & \multicolumn{2}{|c|}{ Biased correlation } & \multirow{2}{*}{$\frac{\mathrm{PSD}}{E F D D}$} \\
\hline & $E R A$ & $S S I$ & $E R A$ & $S S I$ & \\
\hline$B(\hat{\zeta} / \zeta) \cdot 10^{-3}$ & -17.824 & -6.113 & -21.685 & -13.017 & 3.335 \\
\hline$V(\hat{\zeta} / \zeta) \cdot 10^{-3}$ & 3.237 & 3.617 & 3.282 & 4.144 & 6.898 \\
\hline$M S E(\hat{\zeta} / \zeta) \cdot 10^{-3}$ & 3.555 & 3.654 & 3.753 & 4.314 & 6.909 \\
\hline$B(\hat{f} / f) \cdot 10^{-3}$ & -1.834 & -2.411 & -1.893 & -2.300 & -2.245 \\
\hline$V(\hat{f} / f) \cdot 10^{-6}$ & 0.432 & 5.523 & 0.264 & 2.077 & 14.809 \\
\hline $\operatorname{MSE}(\hat{f} / f) \cdot 10^{-6}$ & 3.798 & 11.334 & 3.848 & 7.383 & 19.848 \\
\hline
\end{tabular}

\section{Aeroelastic simulations of offshore wind turbine tower vibrations}

Numerical predictions of the dynamic response of an $8 \mathrm{MW}$ offshore wind turbine have been computed using the aeroelastic simulation tool, FLEX5 [38]. The dynamic response has been used as input for the damping estimation procedure. FLEX5 provides the modal parameters of the model wind turbine, where the damping ratio is obtained by tuning the Rayleigh proportional damping model, which enables a validation of the method proposed for damping estimation. The simulations included aerodynamic and hydrodynamic loading representing a scenario experienced by offshore wind turbines in non-operating conditions. The aerodynamic loading is modelled by the unsteady blade element momentum (BEM) theory [39]. The wind loading is simulated using a Kaimal spectrum, where the mean wind speed acting on the structure is $2 \mathrm{~m} / \mathrm{s}$, and the blades of the wind turbine are pitched out. It is also assumed in this analysis that the contribution from the aerodynamic damping is small, and hence negligible. 


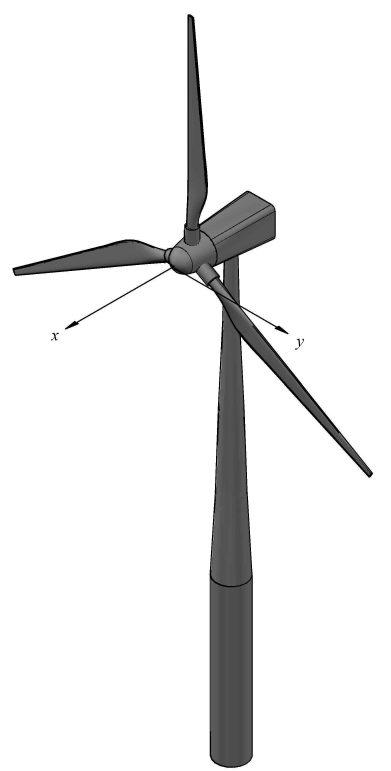

Figure 3: Sketch of an offshore wind turbine on monopile foundation.

The wind turbine is excited by waves heading from 24 different directions based on 2D spectral hindcast data for 100 sea states. It should be emphasized that for most sea states, the wave energy is concentrated around one or a few of these directions. A total of 100 records are retrieved. The unidirectional wave kinematics have been projected to the side-side and fore-aft direction of the wind turbine and the total in-line hydrodynamic loading experienced by the structure is found by Morison's equation. This yields an estimate of the drag, hydrodynamic mass and Froude-Krylov force. The McCamy-Fuchs correction was applied, accounting for diffraction effects. Wheeler stretching has been implemented to extend linear Airy wave theory to provide predictions of the wave kinematics above the mean water level.

The structural model of the wind turbine utilized the principle of virtual work to determine the mass, stiffness and damping matrix. The dynamic character of the system has been represented by a reduced model consisting of two uncoupled modes in the side-side and fore-aft direction. The natural frequency of the first fore-aft mode was $f_{1}=0.234 \mathrm{~Hz}$ with a damping ratio of $\zeta_{1}=0.0127$. The equation of motion has been solved by numerical time integration using a fourth order Runge- 
Kutta scheme with a time increment of $\Delta t=0.1 \mathrm{~s}$, whereby the linear elastic response of the system for 100 sea states has been obtained. The duration of the simulation is 4 hours, corresponding to 3370 periods of the fundamental fore-aft mode.

\subsection{Damping estimates of the fundamental fore-aft and side-side mode}

Results from the aeroelastic simulations of the tower vibrations have been used as input for the automated ERA, COV-SSI, and EFDD identification techniques. Only the dynamic response in the fore-aft and side-side direction at the tower top have been used for the identification. Figure 3 shows a wind turbine, where the fore-aft motion of the turbine is indicated by the $x$-direction and the side-side motion is indicated by the $y$-direction. In practice, the nacelle is equipped with accelerometers, which make tower top vibration measurements readily available. It is therefore of interest to show the potential of only using these measurements for the estimation of damping. The pre-processing of the acceleration time history has been performed as described in the previous section. The identification was automated by the proposed procedure, where the stabilization criteria, the determination of model order and number of blocks in the Hankel and Topelitz matrix were identical to the identification of the 2DOF system described previously in Section 3.

Figure 4 shows the distribution of the estimated damping of the fundamental fore-aft mode obtained by COV-SSI, ERA, and EFDD. The bias, variance and mean squared error of the natural frequency and damping estimates of the fundamental fore-aft mode are further given in Table 2. The probability distribution and errors are obtained by the theoretical damping value $\zeta$ given as input

to the simulation and the estimated value $\hat{\zeta}$. The distribution of the estimated natural frequency and damping ratio of the fundamental side-side mode follows a similar trend, and is therefore not illustrated herein. Additionally the unbiased correlation function estimates lead to a decrease of bias error on the damping estimate, and the least mean squared error. The distribution of the estimates of the biased correlation function are not presented because the deviations from the unbiased correlation function are small. The best estimates are obtained by the unbiased correlation function estimator and the COV-SSI identification procedure. 


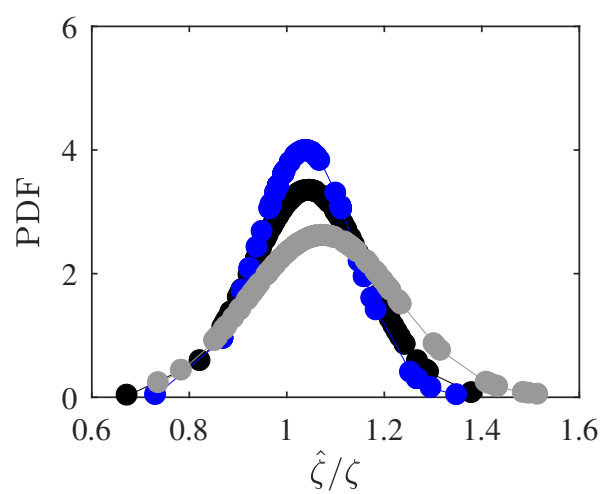

Figure 4: Estimate of the damping of the fundamental fore-aft mode of the wind turbine tower by EFDD (grey bullet), and from from unbiased correlation function estimates by COV-SSI (blue bullet), and ERA (black triangle).

It is noticeable from Table 1 and Table 2 that the estimation errors for damping and frequency increase for the wind turbine tower vibrations, compared to the identification of the equivalent 2DOF system. This increase may occur because the wind turbine tower vibrations are generated by a narrow band process. Another distinction is the positive bias error for the damping estimated by the time domain techniques, where the EFDD clearly has a larger bias on the damping estimates. The latter has been investigated and it was observed that the uncoupling of the modes, obtained by the singular value decomposition in the EFDD procedure, is challenging for modes with closely spaced frequencies, as in the case of the axi-symmetric wind turbine tower shown in Figure 3. It has been recognized that the auto-correlation function obtained in the EFDD, by transforming the singular values to the time domain, for some vibration records exhibit beating. The increase of bias error indicates that simulation models slightly overestimate the damping at certain wind speeds. 
Table 2: The bias, variance and mean squared error of the damping and frequency of the first mode of the fundamental fore-aft mode of the wind turbine tower. The unbiased, biased correlation function estimates and power spectral density are the pre-processing variation for identification techniques, ERA, SSI and EFDD.

\begin{tabular}{|c|c|c|c|c|c|}
\hline & \multicolumn{2}{|c|}{ Unbiased correlation } & \multicolumn{2}{|c|}{ Biased correlation } & \multirow{2}{*}{$\frac{\mathrm{PSD}}{E F D D}$} \\
\hline & $E R A$ & $S S I$ & $E R A$ & $S S I$ & \\
\hline$B(\hat{\zeta} / \zeta) \cdot 10^{-3}$ & 46.062 & 39.832 & 50.141 & 41.003 & 75.010 \\
\hline$V(\hat{\zeta} / \zeta) \cdot 10^{-3}$ & 14.255 & 10.027 & 14.223 & 10.666 & 23.371 \\
\hline$M S E(\hat{\zeta} / \zeta) \cdot 10^{-3}$ & 16.377 & 11.613 & 16.740 & 12.347 & 28.998 \\
\hline$B(\hat{f} / f) \cdot 10^{-3}$ & -18.603 & -18.500 & -1.893 & -18.023 & -16.160 \\
\hline$V(\hat{f} / f) \cdot 10^{-6}$ & 8.052 & 1.210 & 7.946 & 1.162 & 29.266 \\
\hline$M S E(\hat{f} / f) \cdot 10^{-6}$ & 354.118 & 354.355 & 354.694 & 336.437 & 290.419 \\
\hline
\end{tabular}

Although the differences between the various techniques are seemingly minor, the variations specifically in the damping estimates are still important for the design of offshore wind turbines. With respect to applications in the offshore wind turbine industry, the differences between the techniques are crucial in competitive, cost effective and reliable designs, as the experimental findings have an important role for the development, validation and updating of numerical models. The COV-SSI method can be regarded as the method of choice for estimating damping of offshore wind turbines, based on the mean squared error in Table 2. This finding is in agreement with the benchmark technique for output-only estimation of frequency and mode shapes for linear timeinvariant systems [14-17,24]. Suggestions for further improvements of the estimates of damping by covariance driven OMA techniques are discussed at the end of this section.

\subsection{Assessment of robustness of damping estimates}

The estimation of modal parameters may be dependent on the quality of monitored signals and operational conditions, apart from the pre-processing and estimation technique. The operational conditions are regarded negligible for the numerical simulations of the tower vibrations. The quality 
of the damping estimate may deteriorate due to signal noise, too short record periods, excitation level of modes of interest, and the stationarity of the response. The effect on the damping estimates of these four factors has been investigated by the COV-SSI technique, which has been found as the best candidate for the estimation of damping for offshore wind turbines.

\subsubsection{Case 1: Effect of signal noise}

The acceleration records are in practice often contaminated by signal noise, that can increase the variance error of modal parameter estimates. The damping is highly sensitive to the quality of the estimated correlation function, which may be distorted by signal noise. Various noise models exist in literature. Herein the noise is band limited and modeled as Gaussian noise, hence additive zero-mean and white. The addition of white noise has been scaled to the amplitude of the acceleration records, where the amount of signal noise is adjusted by the root-mean-square of the amplitude, and will be referred to as the noise level in percentage. Low noise levels indicate that a small amount of signal noise is added to the vibration record, and high levels indicates that the signal is noise dominant.

The estimates of damping for the fore-aft mode of the wind turbine tower are presented in Figure 5 (a), for variations of noise level from 0-80\%. For low noise levels, the estimates are stable, and unstable for higher levels. A small amount of signal noise increase the randomness of the vibration record, which essentially causes a drop in the variance of the damping estimates, in comparison to identification through a noiseless vibration record.

\subsubsection{Case 2: Effect of measurement duration}

A shortening of the duration of the vibration records yield an increase in the bias and variance error for the correlation function estimates. Figure 5(b) shows the estimates of damping of the fundamental fore-aft mode for various durations of the vibration record. The decrease in the variance error from a $30 \mathrm{~min}$ record to $240 \mathrm{~min}$ record is linear, hence for longer records the estimates of damping improves. The bias error is fluctuating around a mean value, indicating that an increase in the vibration record length may not improve the bias error of the damping estimate. 


\subsubsection{Case 3: Effect of vibration amplitude}

The root-mean-square of the acceleration time history has been considered as an expression for the vibration amplitude. The numerical simulations are representing linear elastic response, and therefore the vibration amplitude does not have an effect on the estimate of damping, see Figure 6(a). Furthermore, the automated procedure is independent of the vibration amplitude. For structures with high amplitude vibrations, the damping ratio may be dependent on the vibration amplitude. However, using an identification procedure driven by the correlation function may not reveal this effect.

\subsubsection{Case 4: Effect of the stationarity of the response}

The assumption of a stationary response in OMA is expected to change over longer periods of time due to operational effects, i.e. the response may become non-stationary due to abrupt changes. Recent developments in time series analysis may overcome them, however it is uncommon to remove such effect and it is not known how the presence of a non-stationary response may affect the damping estimates.

The skewness of the vibration records is shown in Figure 6(b). The mean values of the skewness are $-2.43 \cdot 10^{-4}$ and $-7.32 \cdot 10^{-4}$ in the fore-aft and side-side direction respectively, indicating a minor negative skewness. The dispersion of the estimates of damping around the shoulders of the distribution $(\mu \pm \sigma)$ have been measured by the kurtosis of the vibration records. For a normal distribution a kurtosis of 3 is expected. Figure 6(c) shows the kurtosis of the wind turbine tower vibration. The mean values of the kurtosis are 3.10 and 3.09 for the fore-aft and side-side direction, respectively. The excess kurtosis is positive, which indicates heavier tails and peakedness relative to the normal distribution. The range of values of kurtosis and skewness covered by the fore-aft motion is larger, due to the rotor being aligned with the wind direction in the simulations. The automated procedure proposed for identification is not sensitive to small changes of the stationarity of the vibration records, as no clear trends in the damping estimates with the kurtosis and skewness can be observed from the wind turbine tower vibrations in standstill. 
(a)

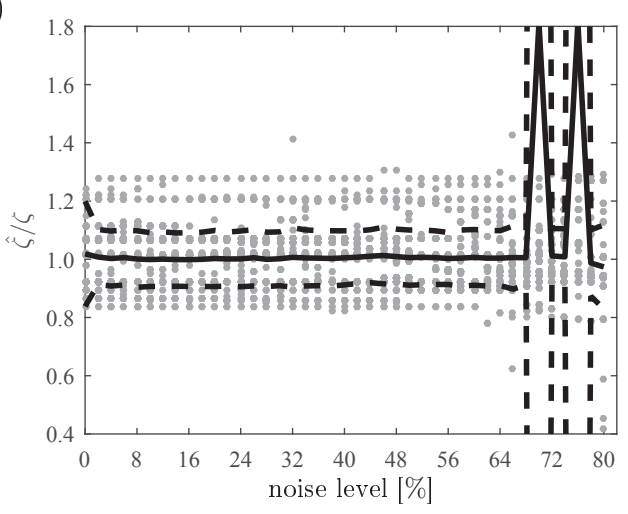

(b)

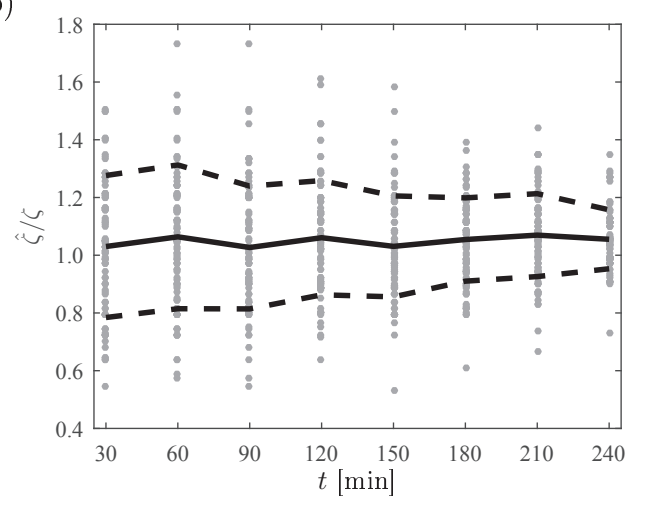

Figure 5: Estimates of the damping of the fore-aft mode (grey), mean of the estimates (black) and variance of the estimates (dotted black) for, (a) variations of noise level and (b) measurement time.

(a)

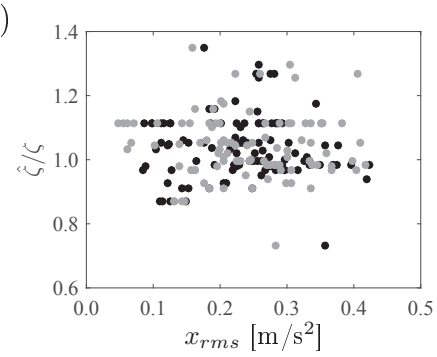

(b)

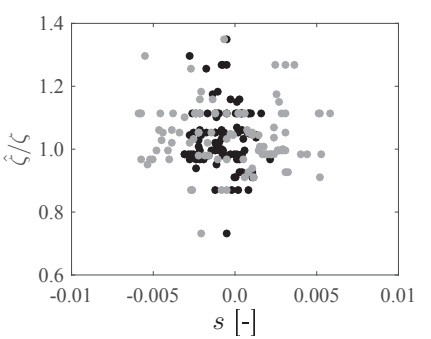

(c)

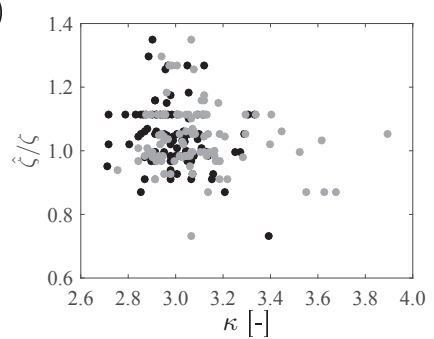

Figure 6: Estimates of damping of the fundamental fore-aft mode (grey) and side-side mode (black) for variations of (a) root-mean-square, (b) skewness and (c) kurtosis of the acceleration time history.

\subsubsection{Discussion on improvements of the estimation technique}

Any estimated structural model is only accurate to a certain level, because the complex behavior of real systems is not adequately represented by the assumed model. It has been highlighted that the errors have two sources, which are the bias and the variance. This study suggests that reduced bias of the estimates of damping for ambient vibrations of offshore structures can be obtained by developing techniques suitable for extracting vibrations which are caused by white noise excitation, 
rather than a narrow banded excitation source.

Bias error may also be reduced by allowing increased flexibility of the model order to obtain a correct description of the system. Reduced bias error can however be at the cost of increased variance error. Therefore there is a need for tuning of the bias-variance trade-off. Tuning can be preformed by minimization of the mean square error. The trade-off may be governed by the model order, which in this study has been restricted by the the stabilization criteria.

In this context it is important to highlight that an error fit, also known as overfitting, may be introduced. Overfitting can be caused by i.e. allowing a high model order. The model order selection rules with respect to estimation of damping may have convexity issues and can therefore lead to multiple optimal model orders. Evolutionary minimization algorithms can be applied, but may not show success. Therefore it is suggested that regularization techniques should be considered in order to avoid overfitting and eventually further improve tuning of bias-variance trade-off for estimation of damping.

\section{Vibration monitoring of offshore wind turbine tower during standstill}

The COV-SSI method has been identified as the most effective damping estimation technique. This method has therefore been used for the estimation of modal parameters based on vibration records from an actual $8 \mathrm{MW}$ offshore wind turbine in non-operating conditions. It is the aim of this section to demonstrate the effectiveness of the proposed automated OMA technique for estimation of damping from the tower top accelerations. The vibration records consist of 12 acceleration time histories of 2 hours duration. During standstill the blades were pitched out. Measured 10 min mean wind speed at the nacelle was fluctuating between $7.5 \mathrm{~m} / \mathrm{s}$ and $12 \mathrm{~m} / \mathrm{s}$. A triaxial accelerometer was mounted on the tower, $7 \mathrm{~m}$ below the center of gravity $(\mathrm{CoG})$ of the nacelle, indicated as second level in Figure 7. Vibrations were also monitored at the first level, see Figure 7, but will not be utilized in the present analysis. The wave conditions were not monitored. 


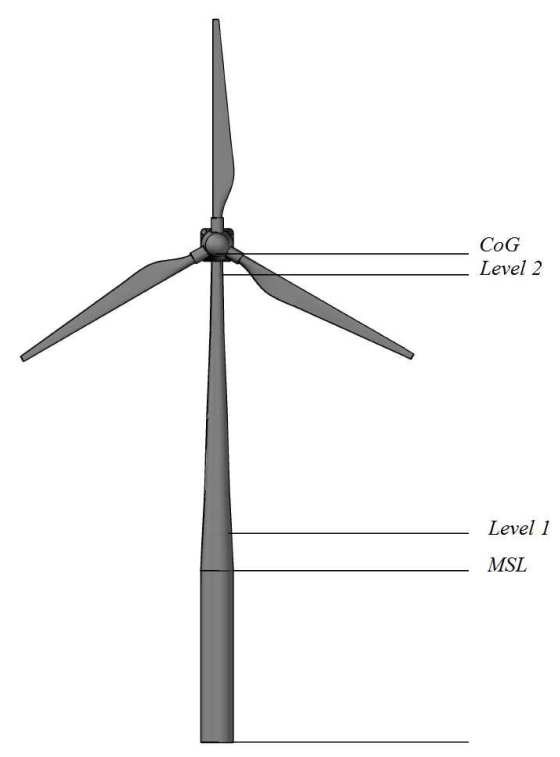

Figure 7: Sketch of the front view of an offshore wind turbine indicating the accelorometer locations at level 1 and 2 , the center of gravity (CoG) and mean sea level (MSL).

An acceleration time history is shown in Figure 8(a), which shows that the amplitude in the fore-aft direction is greater than in the side-side direction for that record. The acceleration level is however dependent on the wave height and the main wave direction. The accelerations were recorded with a sampling frequency of $10 \mathrm{~Hz}$, corresponding to $\Delta t=0.1 \mathrm{~s}$ as in the previous numerical case study. The PSD of the acceleration records, shown in Figure 8(b), confirms that the side-side direction is less excited than the fore-aft direction. The fundamental mode at approximately 0.23 $\mathrm{Hz}$ is mostly excited as indicated by the figure. 
(a)

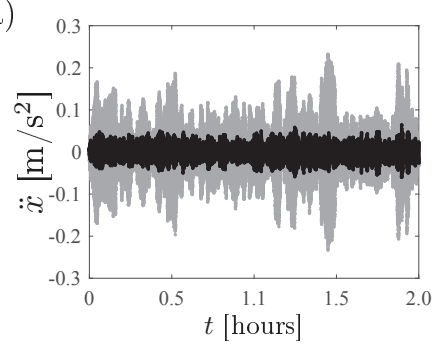

(b)

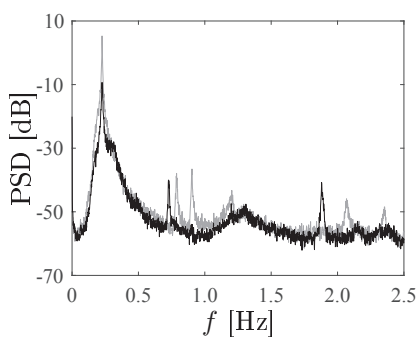

(c)

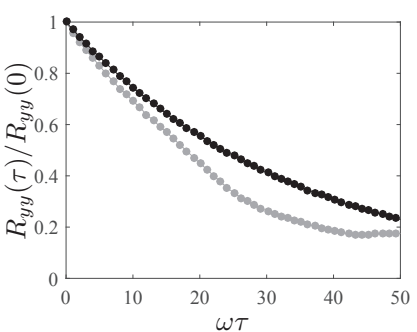

Figure 8: Example of the (a) acceleration time history and (b) power spectral density of the fore-aft motion (black) and side-side motion (grey) of the wind turbine tower at stadstill. (c) The maxima of the envelope of the autocorrelation function (black) fitted to the envelope of the impulse response function of the estimated parameters (grey) by COV-SSI.

\subsection{Damping estimates of the fundamental fore-aft and side-side mode from monitoring data}

The automated COV-SSI procedure has been used to estimate the damping ratio of the fundamental fore-aft and side-side mode during a 24 hour monitoring period. No filtering has been applied to the acceleration records and the mean value has been subtracted from the acceleration to adjust for the assumption of a zero mean process. The pre-processing has been performed for the unbiased correlation function, which has been found to result in a least bias error on the damping estimate. The stabilization of poles is shown in Figure 9 for a suitable range of model orders. As in the previous sections the criterion in (12) has been set to $0.5 \%$ for stabilization in both frequency and damping. Figure 8 (b) shows that pairs of distinct poles appear approximately at $0.23 \mathrm{~Hz}, 1.2 \mathrm{~Hz}$ and $2.2 \mathrm{~Hz}$. The automated system identification procedure determines the lowest model order, at which the poles are stabilized in frequency and damping. The number of lags included in the estimation procedure has been varied and for the considered acceleration records the number of time lags fluctuated around 150, corresponding to 3.5 periods of the fundamental fore-aft mode. The number of periods determined by the automated procedure from the monitoring data is lower than what was determined for the identification of the wind turbine tower from numerical simulations. The numerical simulations, discussed in the previous section, are ideal realizations of a stochastic response of a wind turbine tower in standstill. The fit of the auto-correlation with free decay computed by 
the identified parameters is illustrated in Figure 8(c). The resulting estimates of damping and the natural frequency of the fundamental side-side and fore-aft mode are shown in Figure 10(a)-(b) for the full 24-hour monitoring period.

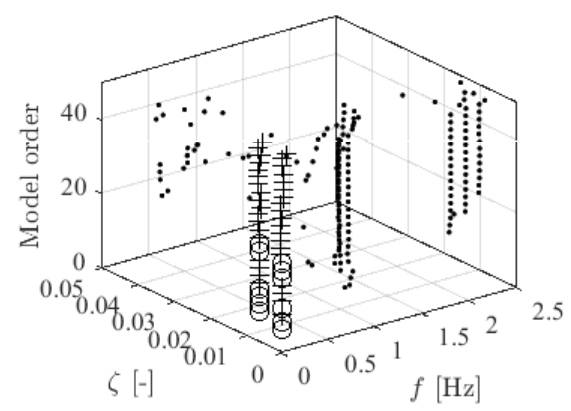

Figure 9: Stabilization diagram of the damping and frequency of the wind turbine tower vibration, (circle) pole stabilized in damping and frequency, (cross) pole stabilized in frequency, (dot) unstable pole.

The mean fundamental natural frequency of the side-side motion and fore-aft motion are, $0.227 \mathrm{~Hz}$ and $0.226 \mathrm{~Hz}$ respectively. The fundamental natural frequency associated with the fore-aft motion is consistently lower than the fundamental natural frequency obtained from the side-side motion. The mean damping of the fundamental side-side mode is 0.0121 . The mean damping of the fundamental fore-aft mode is $42 \%$ lower relative to the side-side mode, with a value of 0.0070 .

For an offshore wind turbine in non-operating conditions it would be expected that the damping of the side-side mode is higher than for the fore-aft mode, because the blades are pitched out. This causes a larger blade surface to interact with the surrounding wind flow in the side-side direction and therefore the aerodynamic damping is more dominant. The aerodynamic drag force is controlled by the pitch angle. The difference between the damping of the fore-aft and side-side mode is therefore dependent on the wind veloctiy and the pitch angle. The opposite tendency is expected for an offshore wind turbine in operation, namely that the damping of the side-side mode is lower than for the fore-aft mode due to aerodynamic damping. During operation the position of the nacelle is controlled such that it is aligned with the main wind direction, which results in more aerodynamic 
damping in the fore-aft direction than in the side-side direction. The contribution to damping from the interaction of the water and monopile foundation is not expected to be a governing factor for the observed difference in damping. The variation of hydrodynamic damping is mostly expected to depend on the wave height and period. The main wave direction may vary and as a result cause slight variations of the added damping to the fore-aft and side-side mode. The differences are not expected to be significant since the hydrodynamic drag force, which dominates the damping mechanism, does not change with wave direction for an axis-symmetric monopile foundation.

The estimates of the frequency and damping show variations during the monitoring period. The observed variations of the natural frequency can be attributed to the variation in the level of vibration amplitude. A relation has been found between the root-mean-square (RMS) of the resulting acceleration time history and the natural frequency. Figure 10(c) shows how the natural frequency decreases slightly when the RMS value increases. This decrease is assumed to be caused by two factors: The additional hydrodynamic mass induced by an increase in water level and the variation of stiffness with respect the level of vibration amplitude.

The variation observed in damping does not appear to be related to the vibration amplitude or changes in the excitation level. It is suggested that the variation in damping is related to the variation in wind speed and one or a combination of the following factors related to the estimation and measurements: The signal noise, the record length, and the bias and mean square error of the estimation technique. Further investigation should be conducted, to isolate the effects of these factors, and thereby understand the variability of the damping estimates. 
(a)

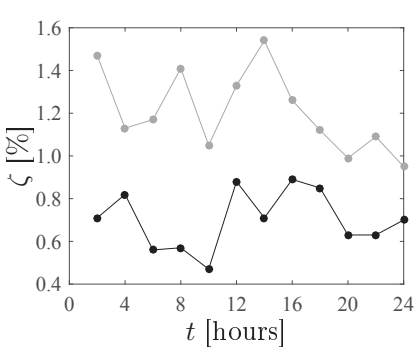

(b)

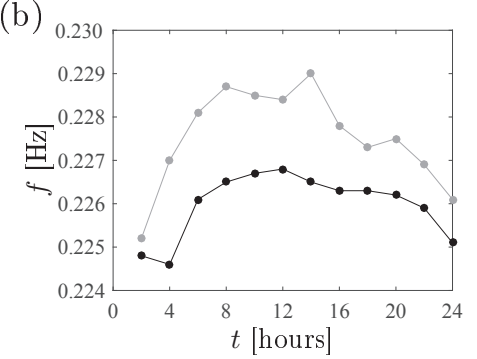

(c)

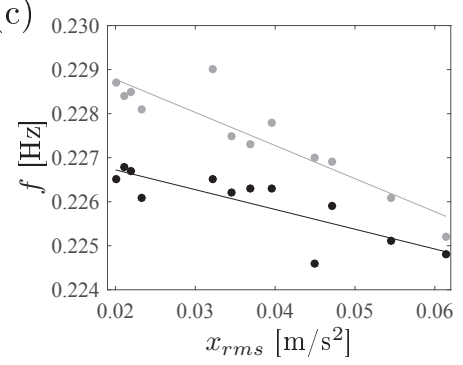

Figure 10: COV-SSI identification of (a) damping and (b) natural frequency of the fundamental fore-aft mode (black) and side-side mode (grey) during a 24-hour monitoring period of the wind turbine tower in standstill, and for (c) variations of the root-mean-square of the acceleration records.

\section{Conclusions}

The following conclusions may be drawn from the automated identification of damping from the present study:

- The proposed automated procedure eliminates the need for a user interference for the selection of model order and size of the block-Hankel and block-Toeplitz matrices.

- The bias error on the damping estimates by ERA and COV-SSI is negative for broad band inputs, and of larger and positive magnitude for narrow banded inputs.

- The COV-SSI is found to be more effective in damping estimation, highlighting a lower mean squared error in comparison to the error from the ERA and the EFDD.

- High signal to noise ratios disturbs the COV-SSI identification leading to higher variance errors on the damping estimates.

- Increase in the vibration record length reduces the variance error on the damping estimate, obtained by the COV-SSI. 
- The fundamental fore-aft and side-side mode of an offshore wind turbine in non-operating conditions have closely spaced natural frequencies, which results in discrepancies in the estimate of damping, and consequently introduces beating for the EFDD technique.

The estimated damping of the fundamental fore-aft tower mode is found to be considerably lower than the damping of the fundamental side-side mode, during non-operating conditions of an offshore wind turbine. Additionally, it is lower than estimates found from numerical simulations for the wind turbine under identical conditions. This emphasizes the importance of reliable estimates of damping for development of aerodynamic models and subsequently reliable prediction of the lifetime of wind turbine structures.

\section{Acknowledgements}

The authors gratefully acknowledge the helpful discussions with Niels Jacob Tarp-Johansen from Dong Energy.

\section{References}

[1] Gao Z, Ding SX and Cecati C. Real-time fault diagnosis and fault-tolerant control. IEEE Transactions on Industrial Electronics 2015; 62(6): 3752-3756.

[2] Tarp-Johansen NJ, Andersen L, Christensen ED, Mørch C, Frandsen S and Kallesøe B. Comparing sources of damping of cross-wind motion. In: European Offshore Wind 2009: Conference \& Exhibition, Stockholm, Sweden, 14-16 September, 2009.

[3] Damgaard M, Ibsen LB, Andersen LV, and Andersen JK. Cross-wind modal properties of offshore wind turbines identified by full scale testing. Journal of Wind Engineering and Industrial Aerodynamics 2013; 116: 94-108.

[4] Shirzadeh R, Devriendt C, Bidakhvidi MA and Guillaume P. Experimental and computational damping estimation of an offshore wind turbine on a monopile foundation. Journal of Wind Engineering and Industrial Aerodynamics 2013; 120: 96-106. 
[5] Devriendt C, Weijtjens W, El-Kafafy M and De Sitter G. Monitoring resonant frequencies and damping values of an offshore wind turbine in parked conditions. Renewable Power Generation, IET 2014 8(4): 433-441.

[6] Koukoura C, Natarajan A, and Vesth A. Identification of support structure damping of a full scale offshore wind turbine in normal operation. Renewable Energy 2015; 81: 882-895.

[7] Hu WH, Thöns S, Rohrmann RG, Said S and Rü cker W. Vibration-based structural health monitoring of a wind turbine system. Part I: Resonance phenomenon. Engineering Structures 2015; 89: 260-272.

[8] Hu WH, Thöns S, Rohrmann RG, Said S and Rücker W. Vibration-based structural health monitoring of a wind turbine system Part II: Environmental/operational effects on dynamic properties. Engineering Structures 2015; 89: 273-290.

[9] Shirzadeh R, Weijtjens W, Guillaume P and Devriendt C. The dynamics of an offshore wind turbine in parked conditions: a comparison between simulations and measurements. Wind Energy 2015 ; 18(10): 1685-1702.

[10] Gao Z, Saxen H, and Gao C. Guest Editorial: Special section on data-driven approaches for complex industrial systems. IEEE Transactions on Industrial Informatics 2013; 9(4): 22102212 .

[11] Magalhães F, Cunha Ã, Caetano E, and Brincker R. Damping estimation using free decays and ambient vibration tests. Mechanical Systems and Signal Processing 2010; 24(5) : 1274-1290.

[12] Ozbek M, Meng F, and Rixen DJ. Challenges in testing and monitoring the in-operation vibration characteristics of wind turbines. Mechanical Systems and Signal Processing 2013; 41(1): 649-666.

[13] Lew JS, Juang JN, and Longman RW. Comparison of several system identification methods for flexible structures. Journal of Sound and Vibration 1993; 167(3): 461-480. 
[14] Pridham BA, and Wilson JC. A study of damping errors in correlation-driven stochastic realizations using short data sets. Probabilistic engineering mechanics 2003; 18(1): 61-77.

[15] Giraldo DF, Song W, Dyke SJ, and Caicedo JM. Modal identification through ambient vibration: comparative study. Journal of Engineering Mechanics 2009; 135(8): 759-770.

[16] Ceravolo R, and Abbiati G. Time domain identification of structures: comparative analysis of output-only methods. Journal of Engineering Mechanics 2012; 139(4): 537-544.

[17] Magalhães F, Cunha Ã, and Caetano E. Online automatic identification of the modal parameters of a long span arch bridge. Mechanical Systems and Signal Processing 2009; 23(2): 316-329.

[18] Reynders E, Houbrechts J, and De Roeck, G. Fully automated (operational) modal analysis. Mechanical Systems and Signal Processing 2012; 29: 228-250.

[19] Neu E, Janser F, Khatibi AA and Orifici AC. Fully Automated Operational Modal Analysis using Multi-Stage Clustering. Mechanical Systems and Signal Processing 2017; 84: 308-323.

[20] Juang JN and Pappa RS. An eigensystem realization algorithm for modal parameter identification and model reduction. Journal of guidance, control, and dynamics 1985; 8(5): 620-627.

[21] Peeters B and De Roeck G. Reference-based stochastic subspace identification for output-only modal analysis. Mechanical Systems and Signal Processing 1999; 13(6): 855-878.

[22] Reynders E. System identification methods for (operational) modal analysis: review and comparison. Archives of Computational Methods in Engineering 2012; 19(1): 51-124.

[23] Ibrahim SR and Mikulcik EC. A method for direct identification of vibration parameters from the free response The Shock and Vibration Bulletin 1977; 47(4): 183-198.

[24] Fukuzono K. Investigation of Multiple Reference Ibrahim Time Domain Modal Parameter Estimation Technique, M.S. Thesis, Dept. of Mechanical and Industry Engineering, University of Cincinnati, 1986. 
[25] Mellinger P, Michael D, and Laurent M. Variance estimation of modal parameters from outputonly and input/output subspace-based system identification.Journal of Sound and Vibration 2016; 379: 1-27.

[26] Brincker R, Ventura C and Andersen P. Damping estimation by frequency domain decomposition. In: Proceedings of the 19th International Modal Analysis Conference (IMAC), Kissimmee, USA, 2001; 698-703.

[27] Shih CY, Tsuei YG, Allemang RJ and Brown DL. Complex mode indication function and its applications to spatial domain parameter estimation. Mechanical Systems and Signal Processing 1988; 2(4): 367-377.

[28] Jakobsen JB and Hjorth-Hansen E. Determination of the aerodynamic derivatives by a system identification method. Journal of Wind Engineering and Industrial Aerodynamics 1995; 57(2): 295-305.

[29] Jenkins MG and Donald GW. Spectral analysis and its applications, Holden-Day, 1968.

[30] Schmidt H. Resolution bias errors in spectral density, frequency response and coherence function measurements, i: General theory. Journal of Sound and Vibration 1985; 101(3): 347-362.

[31] Bendat JS and Piersol AG. Random data: analysis and measurement procedures, Vol. 729, John Wiley Sons, 2011.

[32] Brownjohn JMW. Estimation of damping in suspension bridges. In: Proceedings of the Institution of Civil Engineers-Structures and Buildings 1994; 104(4): 401-416.

[33] Rüdinger F and Tarp-Johansen NJ. Estimation of Natural Frequency and Damping of Offshore WTGs on Monopiles from Measurements of Ambient Vibrations. In preparation, 2016.

[34] Brincker R, Andersen P and Jacobsen NJ. Automated frequency domain decomposition for operational modal analysis. Proceedings of the 25th SEM International Modal Analysis Conference. 2007. 
[35] Allemang RJ, and Brown DL. A correlation coefficient for modal vector analysis. Proceedings of the 1st International Modal Analysis Conference (IMAC) Orlando, USA, 1982; 1: 110-116.

[36] Clough RW and Penzien J. Dynamics of structures. 3rd ed. Berkeley, Computers \& Structures, 1995, p.480.

[37] Bajrić A, Brincker R and Georgakis CT. Evaluation of Damping Using Time Domain OMA Techniques. 2014 SEM Fall Conference and International Symposium on Intensive Loading and Its Effects. Beijing, China, 2014.

[38] Øye S. FLEX4 - Simulation of wind turbine dynamics. In: State of the Art of Aeroelastic Codes for Wind Turbine Calculations, Kongens Lyngby, Denmark, 1996.

[39] Hansen MH, Hansen AD, Larsen TJ, Øye S, Sørensen P and Fuglsang P. Control design for a pitch-regulated, variable speed wind turbine, RisøNational Laboratory, Roskilde, Denmark, 2005. 